\title{
FACIAL NERVE SCHWANNOMA PRESENTING AS MIXED HEARING LOSS, TINNITUS, AND DIZZINESS
}

\author{
Vincenzo Marcelli' ${ }^{1}$, Fabio Piazza ${ }^{2}$ \\ ${ }^{1}$ Vestibular and Audiology Unit, Department of Otolaryngology, ASL-NA1, \\ Ospedale del Mare, Naples, Italy \\ ${ }^{2}$ Department of Otorhinolaryngology-Head \& Neck Surgery, \\ Carlo Poma Hospital (ASST Mantova), Mantua, Italy
}

\begin{abstract}
INTRODUCTION: Facial nerve involvement in skull base tumors is quite a common finding. However, facial nerve tumors are rare. Facial nerve schwannomas can be observed in $75 \%$ of facial nerve tumors. They are slowly growing, benign tumors that can arise from any segment of the facial nerve-from the cerebellopontine angle to the parotid gland. The most common clinical presentation in patients with an intratemporal schwannoma is a slowly progressing facial nerve dysfunction. Less frequently, a fluctuating or a sudden facial nerve weakness can be seen. Hearing loss, tinnitus, and dizziness can also be observed in facial nerve schwannomas. Hearing loss can be conductive, sensorineural or mixed, depending on the size and site of the tumor that can extend into the middle ear or erode the cochlea. Tumors of the internal auditory canal or of the cerebellopontine angle usually lead to a retrocochlear sensorineural hearing loss.
\end{abstract}

CASE PRESENTATION: The authors present the case of a 19 -year-old man suffering from a left-sided hearing loss, tinnitus and dizziness without any facial nerve dysfunction.

RESULTS: The patient underwent computed tomography (CT) scan and magnetic resonance imaging (MRI) with gadolinium to investigate the middle ear and internal auditory canal. A left facial nerve schwannoma, involving the geniculated ganglion, was diagnosed.

CONCLUSION: Even if the most common symptoms of facial nerve schwannomas are facial nerve-related symptoms, we should always keep in mind that hearing-related and equilibrium-related symptoms can be the first presenting symptoms.

Keywords: facial nerve, schwannoma, mixed hearing loss, tinnitus, dizziness, wait-and-see

Address for correspondence:

Fabio Piazza

Department of Otorhinolaryngology-Head \& Neck Surgery

Carlo Poma Hospital (ASST Mantova)

Strada Lago Paiolo 10

46100 Mantua

Italy

e-mail:prof.piazza@hotmail.it

Received: May 19, 2021

Accepted: June 6, 2021

\section{INTRODUCTION}

Facial schwannomas are benign tumors comprising $1.9 \%$ of all intracranial neoplasms and $0.9 \%$ of all intrapetrous tumors (1-4). The geniculate ganglion is frequently involved by these tumors. More than 400 cases have been reported in the world literature so far and still certain management dilemmas exist (5-7). 
Common presenting symptoms are slowly progressing or fluctuating facial nerve weakness, facial nerve palsy that does not improve within a few months, and, less frequently, facial twitching. Hearing loss, tinnitus, and dizziness can also be observed in facial nerve schwannomas. Tumors arising from the geniculate ganglion and from the tympanic or mastoid segment of the facial nerve can extend into the middle ear leading to a conductive hearing loss. Sometimes, they can erode the cochlea leading to a sensorineural hearing loss. Facial nerve schwannomas originating from the internal auditory canal or from the cerebellopontine angle commonly present with a retrocochlear hearing loss. Decision making is not always simple since surgery often leads to unsatisfactory facial nerve outcomes: House and Brackmann grade III is usually the best possible postoperative function after total tumor removal (8). In case of large intracranial life-threatening schwannomas, surgery is commonly indicated, while the wait-andsee policy is indicated in elderly patients with small tumors and a mild or moderate dysfunction. In case of small tumors in young adults, it is very important to find a balance between the preoperative function of the facial nerve and the possible postoperative one. Should the tumor grow or the facial nerve evolve to a moderately severe dysfunction or worse, surgery will be considered. In cases with small tumors with a perfect facial nerve function in young adults, it is not always easy to find a balance between the preoperative function of the facial nerve and the possible postoperative one. Observation, bony decompression, surgical excision, and stereotactic radiosurgery are the current modalities of management in many cranial base surgery centers (1-7).

The clinical presentation and imaging of a 19-year-old man with a facial schwannoma, whose symptoms were characterized by hearing loss, tinnitus, and dizziness without any facial nerve dysfunction, are discussed.

\section{CASE PRESENTATION}

In January 2017 a 19-year-old man was admitted for a 5-year history of hearing loss and a highpitched tinnitus in the left ear, associated with dizziness, mainly generated by movement. The patient underwent pure tone audiometry (PTA), tympanometry and stapedial reflex study, reflex decay test, au- ditory brainstem responses (ABR), vestibular examination, cervical vestibular evoked myogenic potential (cVEMP), and bithermal caloric test. Moreover, the patient underwent computed tomography (CT) and magnetic resonance imaging (MRI).

\section{RESULTS}

Pure tone audiometry (Amplaid 131) showed a normal hearing in the right ear and a mixed hearing loss in the left ear while speech testing showed a left roll-over suggestive of retro-cochlear injury (Fig. 1).

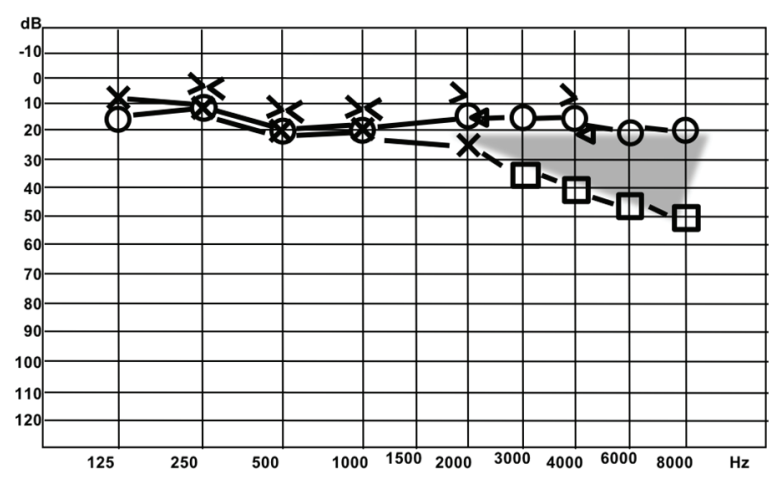

Fig. 1. Pure tone audiometry showing a right normal hearing and a left mixed hearing loss.

Tympanometry (Amplaid 730) showed a normal compliance in the right ear and a very high compliance (out of scale) in the left ear; stapedial reflexes were only partially present with ipsilateral stimulation to the right side (Fig. 2a-2b) and the reflex decay test showed a pathological decay in the left ear with stimulus at $1000 \mathrm{~Hz}$ (Fig. 3).

Auditory brainstem responses were normal in the right ear and absent in the left ear. Evaluation of the tinnitus revealed the presence of a continuous high-pitched ringing $(3.000 \mathrm{~Hz}, 25 \mathrm{~dB})$ in the left ear.

The vestibular examination (infrared videonystagmography-Ulmer VNG, Marseille, France) showed a spontaneous mixed horizontal torsional nystagmus, beating to the right and with the top pole beating toward the right ear, appearing stationary, persistent, partially inhibited by fixation and enhanced by the head-shaking test (9-10). The bithermal caloric test according to Fitzgerald-Hallpike showed a left vestibular deficit equal to $34 \%$ (11). The hyperventilation test showed the transient reversal of the spontaneous nystagmus direction (12-13). The 

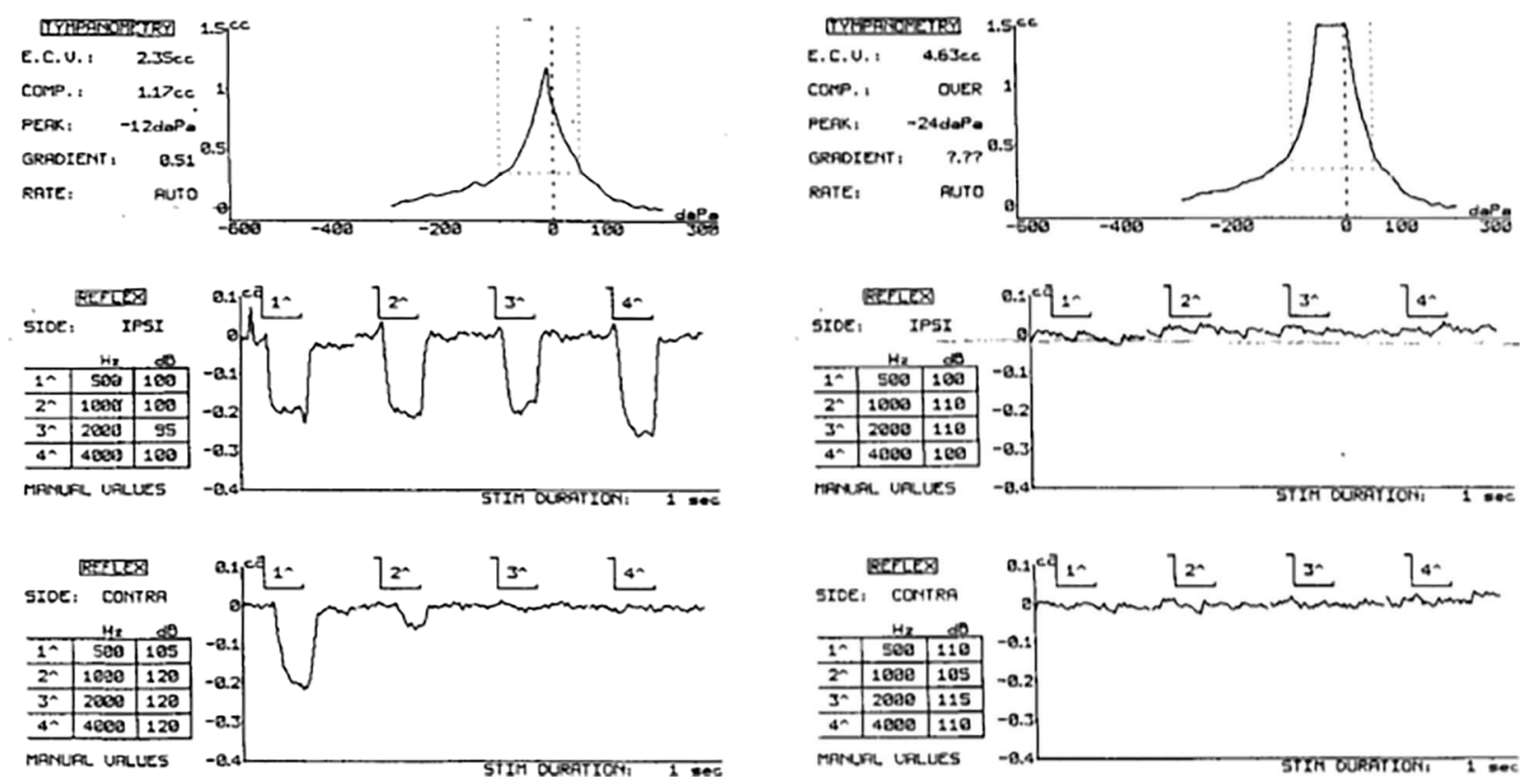

Fig. 2a-2b. Tympanometry showing normal compliance in the right ear and very high compliance in the left ear; partially present stapedial reflexes with ipsilateral stimulation to the right.
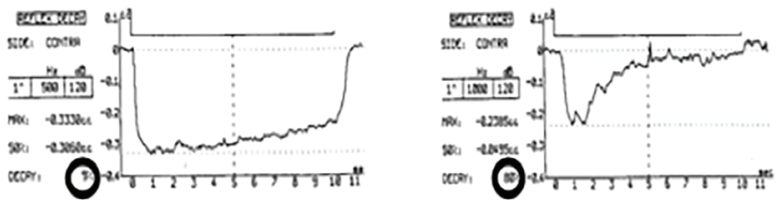

Fig. 3. Reflex decay test showing a pathological decay in the left ear.

clinical head impulses in the horizontal plane (14) showed a left vestibular deficit.

The patient underwent CT scan (Fig. 4) and MRI with gadolinium (Fig. 5) to investigate the left middle ear and internal auditory canal. A left facial nerve schwannoma involving the geniculated ganglion was diagnosed.

\section{DISCUSSION}

The authors documented a left mixed hearing loss and a vestibular deficit, with temporary recovery of vestibular function on the hyperventilation test, demonstrating that a vestibular function was still present. On this basis, the authors hypothesized the presence of a lesion affecting the middle ear (based on the conductive component of hearing loss) and the internal acoustic canal (based on the sensorineural component of hearing loss and on the vestibular
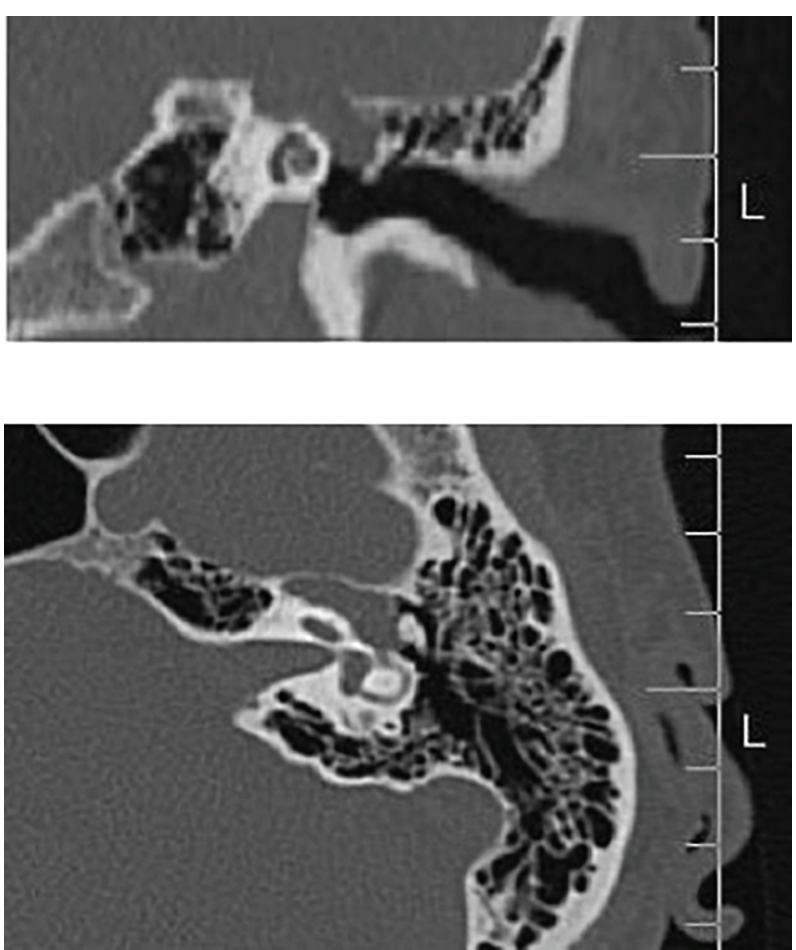

Fig. 4. CT: the tumor erodes the geniculate ganglion and extends into the middle ear.

deficit). The CT scan and MRI confirmed the presence of a left facial nerve schwannoma involving the geniculated ganglion. 


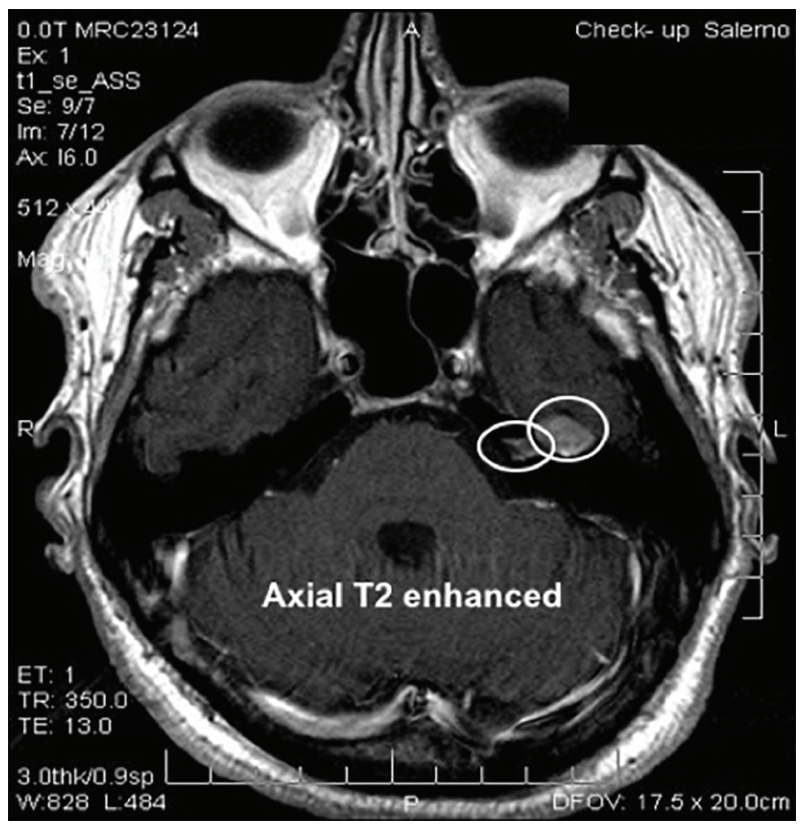

Fig. 5. MRI: the tumor involves the geniculate ganglion and the fundus of the internal auditory canal.

Considering that the symptoms were rather mild and that there was no facial nerve dysfunction, the patient refused surgery and gamma knife treatment, preferring the wait-and-see policy. Up to the present moment, serial MRI has shown no increase of the tumor size; the audiological and vestibular symptoms are stable and the facial nerve function is still perfectly normal.

Even if the most common symptoms of facial nerve schwannomas are facial nerve-related symptoms, we should always keep in mind that hearingrelated and equilibrium-related symptoms can be the first presenting symptoms. Facial nerve schwanno$\mathrm{ma}$ is a condition with a certain risk of misdiagnosis and hearing loss (15). Full audiological assessment should be carried out.

Diagnosis of facial nerve schwannomas relies on CT and MRI. Both are necessary to precisely assess tumor size and extension. CT scans are particularly useful to examine the intratemporal facial nerve, since it can show the erosion of the bony canal of the facial nerve and the presence of a soft-tissue mass in the middle ear. MRI is complementary to CT: it allows the evaluation of the intratemporal and extratemporal extension of the tumor.

\section{CONCLUSION}

A facial nerve schwannoma confined within the cerebellopontine angle can be difficult to distinguish from a vestibular schwannoma: the presence of facial nerve dysfunction is a rare finding in vestibular schwannomas. Nevertheless, the absence of facial nerve weakness is not sufficient to rule out the diagnosis of a facial nerve schwannoma.

The wait-and-see policy is appropriate in patients with perfect facial function and mild hearing and/or equilibrium dysfunction.

\section{Acknowledgement}

We are grateful to Prof. Antonella Piazza for her substantial support and contribution to this work.

\section{Conflict of Interest:}

No grants supported the work.

The authors declare the absence of conflicts of interest.

\section{REFERENCES}

1. Nadeau DP, Sataloff RT. Fascicle preservation surgery for facial nerve schwannomas involving the posterior cranial fossa. Otol Neurotol 2003;24(2):317-25. doi: 10.1097/00129492-200303000-00031.

2. Fagan PA, Misra SN, Doust B. Facial neuroma of the cerebellopontine angle and the internal auditory canal. Laryngoscope. 2003;103(4 Pt 1):442-6. doi: 10.1002/lary.5541030414.

3. Lassaletta L, Castro A, Patrón M, Sarriá MJ, Gavilán J. Diagnosis of intracranial facial schwannoma: clinical, and radiological factors, and the value of immunohistochemistry. Acta Otorrinolaringol Esp. 2004;55(9):399-403. doi: 10.1016/ S0001-6519(04)78543-x.

4. Sherman JD, Dagnew E, Pensak ML, Van Loveren HR, Tew JM. Facial nerve neuromas: report of 10 cases and review of the literature. Neurosurgery. 2002;50(3):450-6. doi: 10.1097/00006123-20023000-00004.

5. Kumar A, Deopujari C, Shah A. Facial schwannomas: Various presentations and their management with literature review. Indian J Otolaryngol Head Neck Surg. 2013; 65(Suppl 3): 670-5. doi: 10.1007/ s12070-011-0397-1.

6. Prasad SC, Laus M, Dandinarasaiah M, Piccirillo E, Russo A, Taibah A, et al. Surgical management 
of intrinsic tumours of the facial nerve. Neurosurgery. 2018;83(4):740-52. doi: 10.1093/neuros/nyx489.

7. Bacciu A, Nusier A, Lauda L, Falcioni M, Russo A, Sanna M. Are the current treatment strategies for facial nerve schwannoma appropriate also for complex cases? Audiol Neurootol. 2013; 18(3):184-91. doi: 10.1159/000349990.

8. House JW, Brackmann DE. Facial nerve grading system. Otolaryngol Head Neck Surg. 1985;93(2):146-7. doi: 10.1177/019459988509300202.

9. Takahashi S, Fetter M, Koenig E, Dichgans J. The clinical significance of head-shaking nystagmus in the dizzy patient. Acta Otolaryngol. 1990; 109(12):8-14. doi: 10.3109/00016489009107409.

10. Tseng HZ, Chao WY. Head-shaking nystagmus: a sensitive indicator of vestibular dysfunction. Clin Otolaryngol Allied Sci. 1997;22(6):549-52.

11. Fitzgerald G, Hallpike CS. Studies in human vestibular function: I. Observations on the directional preponderance of caloric nystagmus resulting from cerebral lesions. Brain. 1942; 65(2):115-37. doi: 10.1093/brain/65.2.115.
12. Califano L, Melillo MG, Vassallo A, Mazzone S. Hyperventilation-induced nystagmus in a large series of vestibular patients. Acta Otorhinolaryngol Ital. 2011; 31(1):17-26.

13. Sakellari V, Bronstein AM, Corna S, Hammon CA, Jones S, Wolsley CJ. The effects of hyperventilation on postural control mechanisms. Brain. 1997; 120(Pt 9):1659-73. doi: 10.1093/brain/120.9.1659.

14. Halmagyi GM, Curthoys IS. A clinical sign of canal paresis. Arch Neurol. 1988; 45(7):737-39. doi: 10.1001/archneur.1988.00520310043015.

15. Li Y, Dai C. A retrospective study on facial nerve schwannomas: a disease with a high risk of misdiagnosis and hearing loss. Eur Arch Otorhinolaryngol. 2017; 274(9):3359-66 doi: 10.1007/ s00405-017-4665-X. 